\title{
The CogMedia Project: Open Data and Tools for Linking Cognitive Science and Mass Media
}

\author{
Rick Dale \\ Department of Communication \\ University of California, Los Angeles \\ co-mind.org/rick, rdale@ucla.edu \\ (unpublished draft, v1.1; feedback, criticism welcomed)
}

August 11, 2020

\begin{abstract}
Minds produce and consume news media. Because of this central relevance of minds, theories of mass media may be informed by the methods and measures of cognitive science. These measures could enhance understanding of the factors guiding news consumption habits. These measures could also help to explain how news themes accumulate collective attention. CogMedia is introduced here as an open resource for facilitating a connection between mind and mass communication. It contains hundreds of thousands of major headlines from large news media organizations, primarily in the United States. In addition, it has a set of open analysis tools for conducting quantitative analysis in R. This paper motivates CogMedia with background theoretical and empirical review. Theoretical discussion centers on complexity science as a framework for understanding mass-scale systems and the cognitive agents that underlie them. Following this background review and motivation, the paper introduces CogMedia, and illustrates potential quantitative analysis and data visualization using these open tools. Future empirical work is discussed, along with relevant theoretical implications.
\end{abstract}

Keywords: cognition, news media, complex systems, dynamics, big data, open data, web application

\section{Introduction}

Media emerge from minds. In turn, minds are shaped by these media. This is a relationship of mutual influence. Consider a plausible example. The attention of modern Internet readers may crave efficient sports-like rankings: "19 Reasons Why There's Absolutely Zero Reason To Not Wear A Mask If You Can," ${ }^{1}$, "19 Of The Best Places To Camp In The US If You're Craving Nature" ${ }^{2}$ or even "The 33 Most Important Lists Ever Written." 3 This craving may reflect a long-standing quality of human information consumption (Young, 2017), now amplified by the qualities of recent media (Carr, 2020). A media company can capitalize upon this niche, producing such lists and related viral media as a primary offering (Tandoc Jr \& Jenkins, 2017; Young, 2017). These viral offerings serve as a communication strategy for the company. They also serve as a cognitive lens for readers, perceiving their world through this format. And as readers become familiar with this format, they create yet more demand for it.

This relationship between the media company and readers initiates a positive feedback loop (Meadows, 2008). If sustained, readers might come to see their world as a mass collection of top-10 designations, or kitschy memetic formulations (Young, 2017). The media company, riding this wave of consumption, might grow rapidly in influence, fusing this novel approach with dimensions of traditional media (Tandoc Jr \& Foo, 2018). A

\footnotetext{
${ }^{1}$ https://www.buzzfeed.com/daves4/no-excuses-to-not-wear-a-mask

${ }^{2}$ https://www.buzzfeed.com/kirbybeaton/camping-during-covid-destinations-us

${ }^{3}$ https://www.buzzfeed.com/summeranne/the-33-most-important-lists-ever-written
} 
parallel may be drawn with concepts from evolutionary biology: This cognitive zeitgeist of consumers and the communication strategies of media organizations are in a process of mutual niche construction (cf. Contreras Kallens, Dale, \& Smaldino, 2018; Odling-Smee, Laland, \& Feldman, 2013). Each must adapt to the other and thus each shapes the other.

A conceptual framework based on complex systems may be well suited to understanding this mutuality. Complexity science and related disciplines investigate how mass-scale systems emerge collectively from the actions of individuals. These disciplines also describe how collective systems feedback onto and thereby shape the individuals that underlie them. This recurrent dynamic, across levels of organization of a complex system, is a natural feature of multiscale systems of all sorts (Bar-Yam, 2004; Bechtel, 2017; Haken, 1987; Laughlin, Pines, Schmalian, Stojković, \& Wolynes, 2000; Kelso \& Engstrom, 2006; Mitchell, 2009; Turvey, 1990). In the cognitive and communication sciences, this dynamic is important for understanding the relationship between the minds of individuals and their cultural practices and artifacts (e.g., Goldstone \& Janssen, 2005; Gureckis \& Goldstone, 2009; Lupyan \& Dale, 2016; Neuman, Guggenheim, Jang, \& Bae, 2014; Sherry, 2015; Silberstein \& Chemero, 2012; Slater, 2007; Spivey, 2020; Streeck \& Jordan, 2009; Wells et al., 2019). A number of theoretical concepts are relevant to this individual-system relationship. These concepts serve to describe the interdependence between the structure and behavior of a large-scale system, and the processes operating among the entities that make up that system. Do systems emerge from the collective action of their entities? Do larger systems, once formed, have a downward causal influence on their entities? Is there a causal circularity between systems and entities, such that their respective qualities derive from their nonlinear interactivity?

In a discussion of the importance of dynamics and time series analysis, Wells et al. (2019) share notes about assessing complex, entangled properties of communication systems, especially in political communication. Assessing processes over time in datasets that measure change offers insights into the underlying complexity of communication systems and their "communicative actors." These authors aver, however, that "...our theoretical frameworks - as well as our often regression-based empirical techniques - are only beginning to come to terms with this complexity." (p. 4024) Embracing complexity science encourages considering new data (such as data organized in time) and new methods to attempt this disentangling, and more precisely model the relationships, such as directions of influence, across a system and its stakeholders (cf. Sherry, 2015; Port \& Van Gelder, 1995; Neuman et al., 2014; Spivey, 2008; Thelen \& Smith, 1996; Uscinski, 2009; Van Orden \& Stephen, 2012).

A review of these more theoretical topics is outside the scope of the present paper, and there are many useful discussions of these ideas (e.g., Chemero, 2011; Dale, Fusaroli, Duran, \& Richardson, 2013; Juarrero, 2009; Streeck \& Jordan, 2009; Richardson \& Chemero, 2014; Sherry, 2015; Turvey, 2004). Indeed, determining which concepts are relevant to mind-media interdependence, and applying these concepts rigorously, faces the challenge of procuring data and tools rich enough to test them quantitatively. The recent importance of expansive and often ecologically valid datasets ("big data") has been remarked by both communication and cognitive scientists (e.g., among many: Joo, Steinert-Threlkeld, \& Luo, 2018; Hilbert et al., 2019; S. C. Lewis, Zamith, \& Hermida, 2013; Morgan, Shanahan, \& Signorielli, 2015; Neuman et al., 2014; Steen et al., 2018; Goldstone \& Lupyan, 2016; Griffiths, 2015; M. N. Jones, 2016; Paxton \& Griffiths, 2017). Large-scale datasets and analyses permit new insights into the relationships among collective systems and their underlying entities. In the case of mass communication and cognition, for example, data that connect media activity at the mass scale with the attention and responsiveness of the consuming public may permit investigation into the flows between them.

The present paper introduces a new resource to facilitate further investigations of this kind. The "Cognition and Media" (CogMedia) project links large-scale news media data to indices of cognitive processing calculated using applied natural language processing. CogMedia samples news media and social media activity continuously, and stamps a large database with both individual and collective measurements. An underlying goal of the project is to facilitate these linkages between lower- and higher-level organization.

This brief paper introduces CogMedia. In what follows, the CogMedia resource is summarized, along with some research directions being pursued by the author and collaborators. Following summary of CogMedia, the paper situates this resource in wider theoretical context, describing how it resonates with themes of computation and "big data" in both communication and cognitive science. The paper concludes by revisiting these broad theoretical 
Table 1: Sources in CogMedia ordered by name, from about June 2019 to June 2020. Alexa (rank) is a ranking of website popularity. Social score ( $s o c$, see below) is an approximation of the average rate of sharing of stories from that source. Very left (VL) to center (C) to very right (VR) represents partisanship as estimated by Allsides.com.

\begin{tabular}{lllrrr}
\hline \hline & Name & & Alexa & $N$ headlines & soc \\
\hline 1 & ABC News & $\mathrm{L}$ & 369 & 11,484 & 1.76 \\
2 & BBC & $\mathrm{C}$ & 214 & 12,093 & 2.67 \\
3 & Boston Globe & $\mathrm{L}$ & 1,042 & 1,372 & 1.19 \\
4 & Boston Herald & $\mathrm{R}$ & 3,430 & 21,651 & 0.49 \\
5 & Business Insider & $\mathrm{C}$ & 121 & 27,911 & 0.56 \\
6 & Chicago Tribune & $\mathrm{C}$ & 2,431 & 127 & 0.09 \\
7 & CNN & $\mathrm{L}$ & 22 & 35,171 & 1.18 \\
8 & Daily Beast & $\mathrm{VL}$ & 354 & 11,135 & 0.76 \\
9 & Fortune & $\mathrm{C}$ & 920 & 10,662 & 0.40 \\
10 & Fox News & $\mathrm{R}$ & 55 & 11,332 & 2.25 \\
11 & LA Times & $\mathrm{L}$ & 328 & 11,027 & 0.84 \\
12 & NBC News & $\mathrm{L}$ & 176 & 19,386 & 1.14 \\
13 & New York Times & $\mathrm{L}$ & 31 & 33,728 & 1.70 \\
14 & NPR & $\mathrm{L}$ & 146 & 9,036 & 2.37 \\
15 & NY Post & $\mathrm{R}$ & 192 & 20,609 & 1.18 \\
16 & Reason.com & $\mathrm{R}$ & 2,514 & 1,784 & 0.40 \\
17 & Reuters & $\mathrm{C}$ & 324 & 26,470 & 2.38 \\
18 & ScienceDaily & $\mathrm{C}$ & 1,334 & 1,719 & 1.30 \\
19 & The Atlantic & $\mathrm{L}$ & 566 & 5,807 & 0.46 \\
20 & The Economist & $\mathrm{L}$ & 1,389 & 422 & 0.40 \\
21 & The Federalist & $\mathrm{VR}$ & 3,409 & 5,374 & 1.33 \\
22 & The Independent UK & $\mathrm{L}$ & 719 & 30,496 & 0.27 \\
23 & The Nation & $\mathrm{VL}$ & 4,315 & 2,967 & 0.64 \\
24 & USA Today & $\mathrm{C}$ & 102 & 28,223 & 0.71 \\
25 & Wall Street Journal & $\mathrm{R}$ & 160 & 3,504 & 0.92 \\
26 & Washington Post & $\mathrm{L}$ & 72 & 4,909 & 0.84 \\
27 & Washington Times & $\mathrm{R}$ & 1,472 & 30,956 & 0.46 \\
\hline & & & & & \\
& & & & & \\
\hline
\end{tabular}

ideas about complex systems: Cognitive and communication science may be integrated through data-driven study of cognitive systems embedded in their media contexts.

\section{CogMedia database}

The primary component in CogMedia is its database. In addition, some initial pre-processing of media data is conducted as these data are stored in the database. These primary components are summarized here. Preliminary research applications are then illustrated below.

\subsection{Source data}

The CogMedia database is generated from collecting real simple syndication (RSS) news feeds from dozens of major news organizations selected to span the entire political spectrum from very left (e.g., Daily Beast) to very right (e.g., The Federalist) according to Allsides.com. These sources are shown in Table 1. At the time of this writing, the headline database contains approximately 375,000 stories. The database now spans over a year, beginning June 2019. It is expanded automatically throughout each day. For each news agency, the RSS feed for major headlines is pulled into the database multiple times each day. These feeds are free and contain the title and sometimes a summary of each story. CogMedia stores a variety of fields associated with these RSS feeds. These are shown in Table 2 .

A day-by-day visualization of these data is shown in Fig. 1, left panel. The dip in coverage in the middle of the plot in the left panel is likely attributable to the holiday season. 

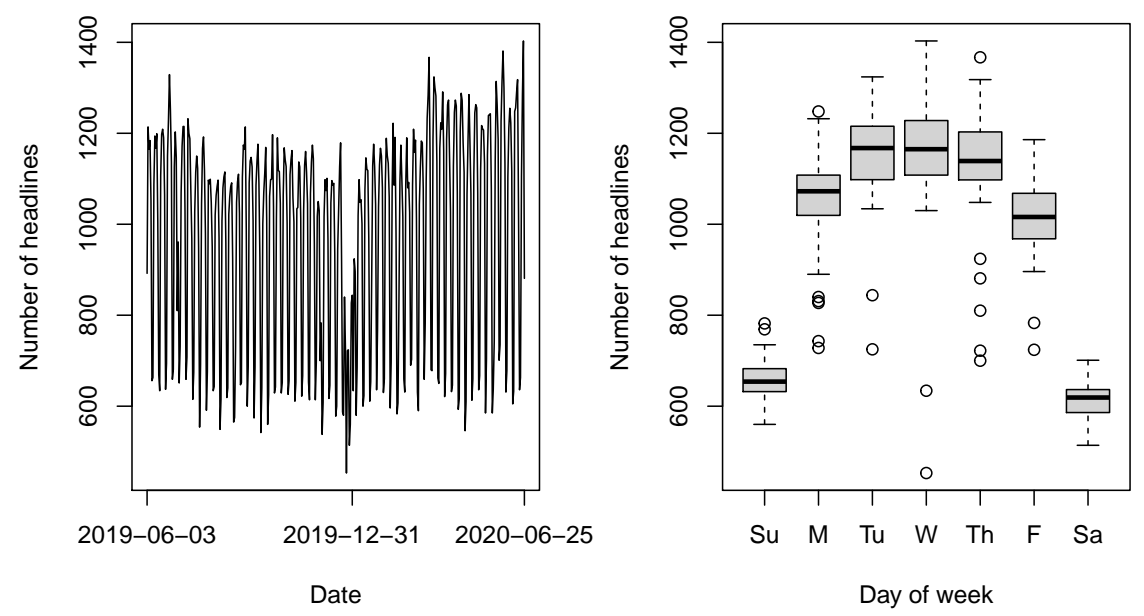

Figure 1: Left panel: Total headlines in CogMedia on each day from June, 2019 to June, 2020. Right panel: Average daily headlines per day of the week, indicating weakly seasonality coverage.

There is clearly a weekday/weekend seasonality, shown in the right panel of Fig. 1. Table 1 shows that sources do vary in the amount of content that they supply. The Economist shares a small selection of stories in its feed, and so occupies only a small percentage of the data. The variation is also due to the schedule of source additions to CogMedia. For example, the Chicago Tribune was just added in June, 2020, and so includes relatively fewer stories than other outlets.

Note also that as a work in progress dependent on sources that may quickly change, CogMedia is in flux. For example, partisan assignment is taken from Allsides.com, and so these scores are subject to their updates (similarly with Alexa rank). In June of 2020, Reuters altered their feed services, so some URLs in the database no longer work. After finding another method to obtain Reuters (using the feeds of Google News), CogMedia now has a relatively higher number of Reuters stories in its database. Finally, while these sources are meant to be raw headline news data, there is the inevitable editorial content that makes its way into the feeds, especially the more partisan outlets.

\subsection{Attention measure: social score}

CogMedia carries out a first-pass automatic analysis of headlines. The primary measure from this process is a social-media sharing score, an approximation of the amount of attention a story immediately receives on Twitter. This is built using Twitter's API from a verbatim search for a story's title. This social score is based on an approximate rate of sharing based on that single sampling: The number of tweets associated with that story divided by the range of time represented in that sample from the Twitter API.

$$
s o c=60^{2} T / \Delta t
$$

Here, $T$ is the total tweets obtained in that single sample, and $\Delta t$ is the time differential between the newest and oldest tweets. The result is in seconds, and so it is converted to tweets per hour, rounded to a single decimal place. The intuition behind this measure is that headlines gaining rapid attention will have more tweets in a shorter range of time. This reduces the time differential, and thus raises soc.

For all stories, this measure is calculated only once shortly after the story is posted on a feed, within approximately 6 hours. It is thus a measure of immediate attention to a story. This score can only be considered an approximation of a true rate of sharing for a story. Reasons for this are obvious. For example, stories with terse titles will lead to a larger sample of tweets, spuriously reflecting a story's interest. In addition, Twitter users may share stories without their titles, sometimes leading to under-sampling. In general, this 
Table 2: Primary fields retrievable from the CogMedia dataset.

\begin{tabular}{lcl}
\hline \hline Field & Type & Description \\
\hline source & string & News outlet. \\
title & string & Title of the headline. \\
description & string & Story description available in RSS feed. \\
url & string & URL to the source and full story (e.g., NYT site) \\
social_score & numeric & $\begin{array}{l}\text { An approximate rate of sharing for the } \\
\text { news item post release (see text). }\end{array}$ \\
partisanship & VL-C-VR & $\begin{array}{l}\text { Allsides.com categorization of the story by } \\
\text { partisan association (very left [VL] to center [C] }\end{array}$ \\
& & to very right [VR]). \\
alexa_rank & numeric & Alexa popularity ranking of main news website. \\
\hline
\end{tabular}

simple sharing metric tends to align with stories that are more widely shared. Indeed, it exhibits the expected inverse correlation with Alexa rank: The better the Alexa rank (i.e., lower numeric value), the higher the sharing, as one would expect more popular websites to gain more attention. From Table 1, the correlation between log-transformed Alexa rank and average social score is approximately $r=-0.46, t(25)=-2.6, p=.017$.

\subsection{Application programming interface (API)}

CogMedia has a rudimentary API, permitting researchers to tap into these data for their own purposes. The API is a wrapper around the httr package in R. The tool also comes with a news-reading function, permitting researchers to playfully explore headlines and summaries in their RStudio console. This is illustrated in lines of code here:

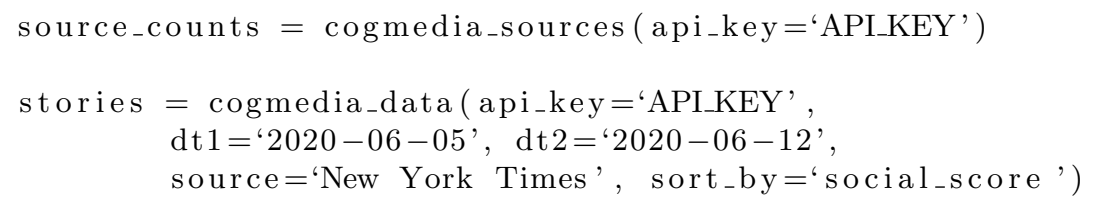

Details about the API can be found in the main CogMedia site: co-mind.org/cogmedia. CogMedia data are also available in batch extractions, by request.

CogMedia includes a function to tag a batch of stories with some elementary natural language processing (NLP) indices (with resources from: Mohammad \& Turney, 2013; Silge \& Robinson, 2016). Users can quickly pull down data from CogMedia and conduct some descriptive analysis of headline trends. First, consider comparing the past week of headlines in the New York Times and Business Insider. Business Insider tends to cover a wide array of matter in its main headline feed, including entertainment and shopping trends. New York Times covers the traditional "hard news" topics. One might predict distinctions in linguistic trends across these outlets. With CogMedia, a quick inspection of this possibility requires a few lines of code.

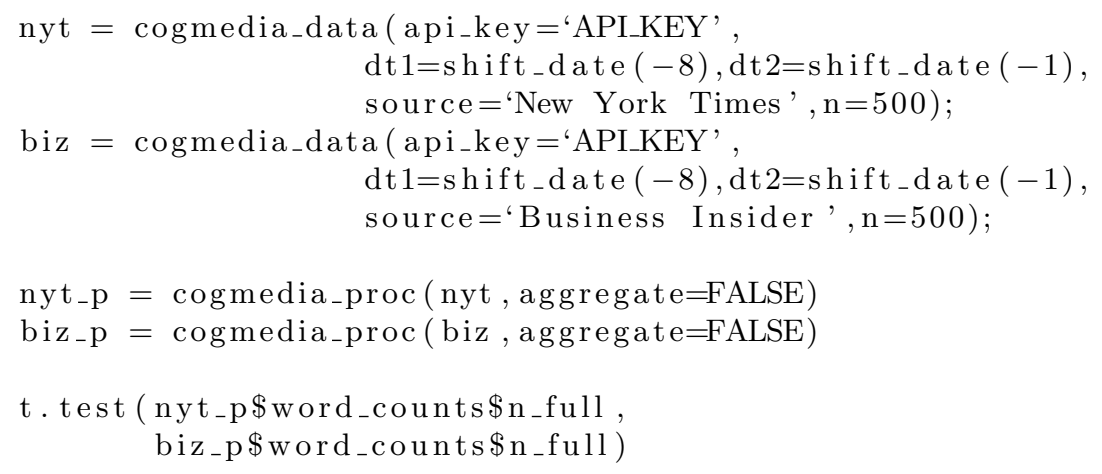

This code extracts the past 500 news stories from these two outlets, and compares them statistically in headline word count. The result of this quick analysis suggests that Business 


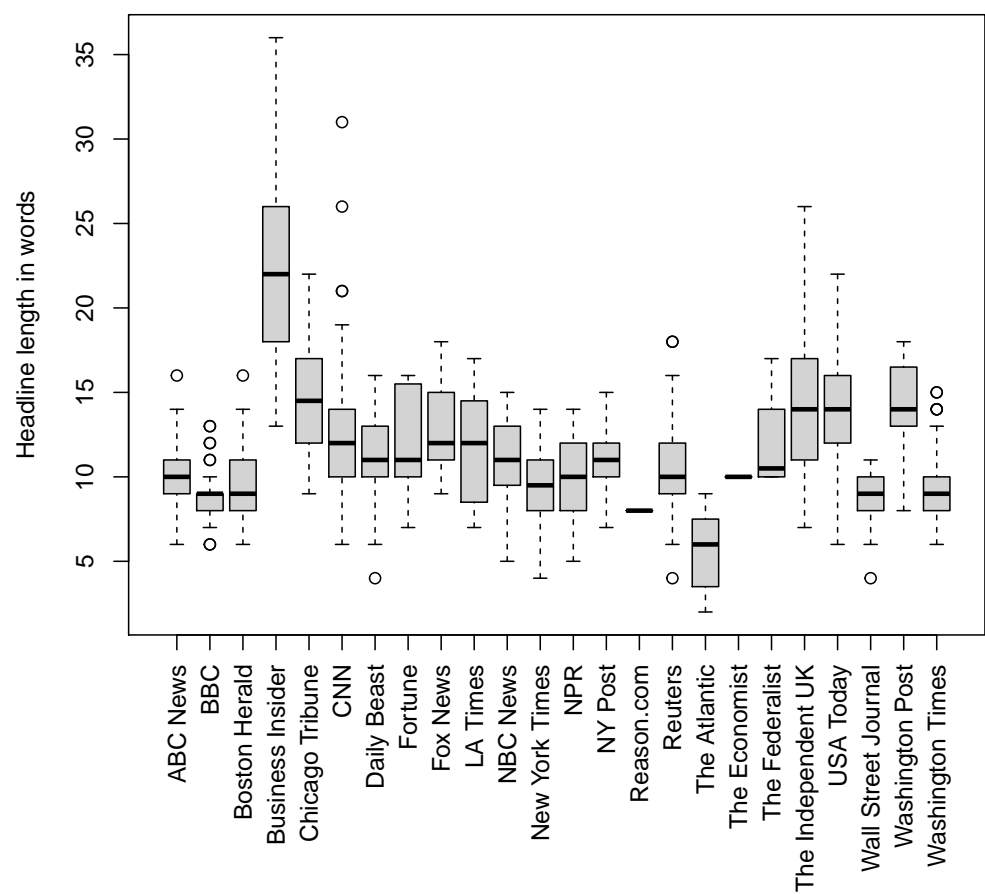

Figure 2: Word counts of recent 1,000 headlines from CogMedia in June, 2020 grouped by source.

Insider has longer average titles in number of words with 20.0, compared to New York Times at 9.3 words, Welch's $t(673.53)=36.8, p<.0001$.

Although this serves as a simple test of the API, prior work has indeed shown that media engagement may be predicted in part by the relative share of civic affairs news (Kernell, Lamberson, \& Zaller, 2018). It seems possible that outlets covering distinct segments of the news may alter their linguistic practices, either strategically or as a consequence of the topic. With "hard news" coverage, it may be more important for a news outlet to aim for cognitively efficient story headlines to maintain attention. Of course to confirm any such result, the analysis requires several inspections of the data and considerations of sampling and so on. These important caveats are discussed below.

These descriptive language statistics are obtained over the past 1,000 news headlines on CogMedia, across all outlets, with a few lines of code in the following way:

$$
\begin{aligned}
& \text { stories }=\text { cogmedia_data( api_key='API_KEY', } \\
& \mathrm{dt}_{1}=\mathrm{shift}_{-} \mathrm{d}_{\text {ate }}(-8), \mathrm{dt}_{2}=\mathrm{sh}_{\mathrm{fft}} \mathrm{d}_{\text {ate }}(-1) \text {, } \\
& \text { source='all', } \mathrm{n}=1000) \text {; } \\
& \text { stories_p }=\text { cogmedia_proc(stories, aggregate=FALSE) }
\end{aligned}
$$

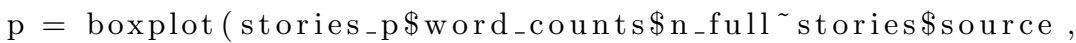

$$
\begin{aligned}
& \text { xaxt }=\text { 'n', xlab =', ylab="Headline length in words') } \\
& \text { axis }(\operatorname{side}=1, \text { at }=1 \text { : length }(p \$ \text { names }), \text { labels }=\text { p } \$ \text { names, } 1 \text { as }=2)
\end{aligned}
$$

The result is shown in Fig. 2. These are elementary illustrations of extracting data from CogMedia's API. Before illustrating some potential applications of CogMedia, some important caveats should be noted. First, sampling issues should be carefully heeded if using the dataset. Different outlets may share different numbers of stories, different subsets of their stories, and distinct sets of topics in these feeds. In addition, the standard bias of time matters in this case too - the prevailing trends in one week may create a distinct effect in headline patterns compared to shifts that occur in a subsequent week. However, these 
issues may offer opportunities for CogMedia to be used as a platform for undergraduate and graduate training. With free data readily available in a free statistical software package (R), CogMedia can prompt discussion of important limitations and confounds in quantitative research in these domains.

\section{Illustrations and future directions}

CogMedia is closely related to a number of news-aggregation projects, especially in the event-data literature, and these agendas are often much larger in scale (e.g., Althaus, Bajjalieh, Carter, Peyton, \& Shalmon, 2020; Leetaru \& Schrodt, 2013; Schrodt, 2010; Ward et al., 2013). For example, the data from GDELT ${ }^{4}$ includes many millions of news stories, and has been used in a variety of applications, including in a related intermedia agenda-setting analysis (Vargo \& Guo, 2017). The Corpus of Contemporary American English $\left(\mathrm{COCA}^{5}\right.$ ) has been very influential in corpus linguistics, and it aggregates full articles so that it contains about a billion word instances (Davies, 2009). In the audiovisual format, the UCLA NewsScape project ${ }^{6}$ has for many years conducted extensive aggregation of television news channels, and is also an order of magnitude larger than the data described here (Groeling, Joo, Lie, \& Steen, 2016; Joo, Steen, \& Turner, 2017).

Despite its relatively smaller size, CogMedia may serve as a volume of data tractable for rapid import and text-based analysis in common software packages. The author and collaborators are exploring how these data may inform investigations of mind-media dynamics described in the introduction. In the next few sections, some potential applications are illustrated. These are preliminary and tentative summaries of early stage research, but illustrate research feasibility with CogMedia data.

\subsection{Cognitive-media linkages}

One way of linking cognitive and media levels of analysis is to explore how measures of cognitive processing predict information spread online. Prior work suggests that the fluency with which minds process information can guide attention, desirability and even aesthetic judgment (Oppenheimer, 2008; Reber, Schwarz, \& Winkielman, 2004). CogMedia helps quantify the ease of processing of a news headline, and this may correlate this with the rate at which a story is initially shared (Luna, Alegria, \& Dale, 2020). The measure of ease of processing is quite simple, and specifies a psycholinguistic "upper bound" of processing time. To measure this upper bound reaction time $\left(R T^{\max }\right)$, data are used from the English Lexicon Project (Balota et al., 2007). This project is based on a large multi-laboratory initiative to get precise mental processing times on individual words. It has resulted in a dataset of over 40,000 individual words in English, and has been extended to other languages.

Each news headlines is indexed with a score, $R T^{\max }$, defined as:

$$
R T^{\text {max }}=\sum_{\text {word }}^{\text {headline }} R T_{\text {word }}
$$

This measure is simply the sum of reaction times associated with the lexical decision time for words making up a title. Sentence processing by the human mind actually involves more complex patterns of chunking (Isbilen \& Christiansen, 2020), in that collections of words, especially frequent ones, tend to be processed together. This suggests that the $R T^{\max }$ measure is a sort of upper bound: It is the processing time required if a cognitive system treats a headline as a list of disconnected words.

Despite its simplicity, this measure has some useful properties. It encapsulates sentence length, because it bears a close linear relationship with word count in headlines. So it naturally captures an informational load measure. However, this linear relationship is approximate, as it depends on the ease of processing of its component words. The variation on this measure may reveal a kind of "cognitive echo" of fluency at the collective scale.

In this project, this $R T^{\max }$ upper bound is examined for how well it predicts social media sharing rate, soc, defined above. It is hypothesized that information load will inversely

\footnotetext{
${ }^{4}$ https://www.gdeltproject.org

${ }^{5}$ https://www.english-corpora.org/coca/

${ }^{6}$ http://newsscape.library.ucla.edu
} 

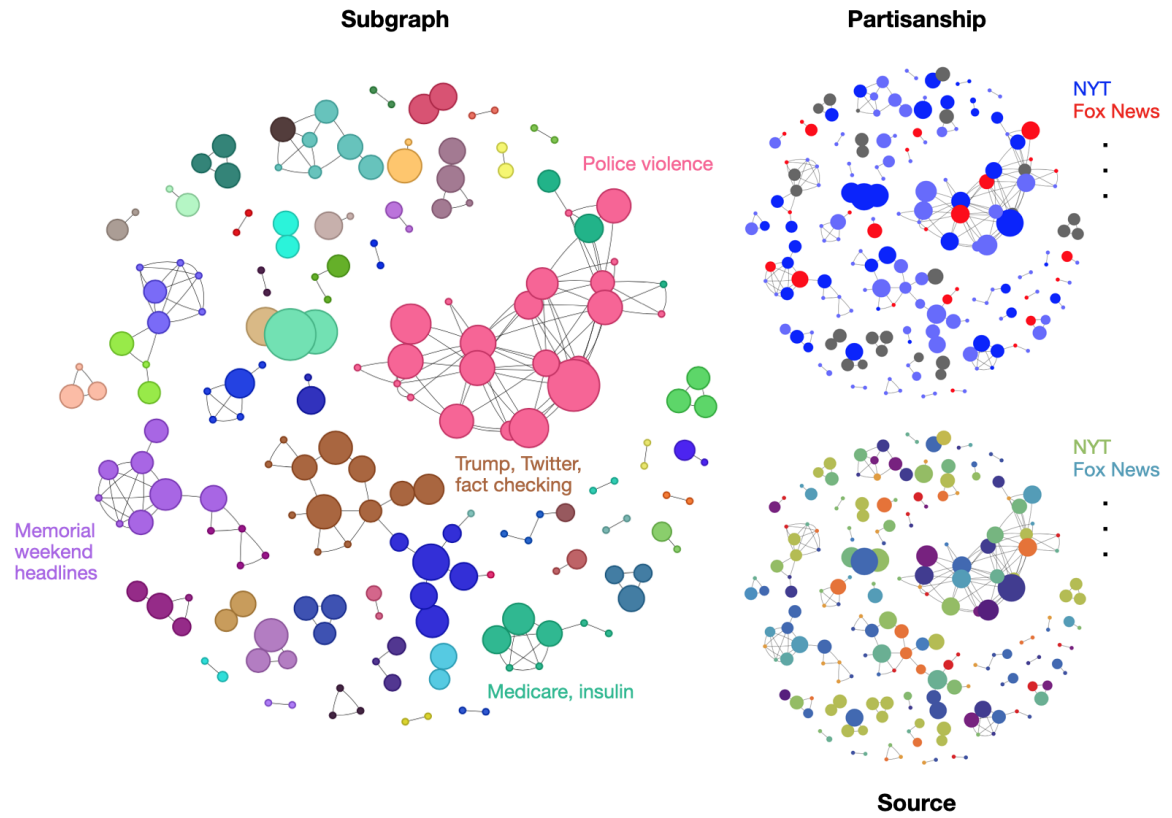

Figure 3: Illustrations of newsgraph network diagrams. Subgraph: Newsgraph from May 26th, 2020 (evening). Each node represents a story, and is connected to other stories that share words. The largest cluster of related news stories emerges from coverage of videos relating to police violence and racism. With this visualization, users can quickly discern distribution of media attention. Size of the node represents how widely each is shared on social media. Color of dots are randomly assigned to make subgraphs of stories easier to find. Partisanship: The same newsgraph with nodes recolored by partisan association (blue $=$ left, gray $=$ center, red $=$ right). Source: The same newsgraph can be recolored to represent source, and permits analysis of intermedia attention - how multiple outlets cover the same news trends. The current newsgraph can be viewed here: co-mind.org/cogmedia/browse/?scr=newsgraph

relate to sharing: The more load, the less sharing. So far analyses yield relatively weak but some significant effects of cognitive processing. Results also suggest these effects may vary by news outlet. Despite such small effects, though, these findings do suggest that cognitive processes may leave a "signature" at the collective scale. In terms consistent with theoretical discussion above: Processing constraints of individual cognitive agents act as a mechanism that amplifies or dampens particular news trends as a function of their ease of processing (see also Steen \& Dale, in preparation).

\subsection{Intermedia attention}

Another application derives from analysis of coverage across news outlets. With a large database that includes many distinct outlets, it is possible to assess what stories are being covered by multiple sources (cf. story chains: Nicholls \& Bright, 2019). This is akin to analyzing media "attention," and patterns in wider news coverage, such as framing and intermedia agenda setting (McCombs \& Reynolds, 2009). It reflects the kind of filtering and focus that news media outlets are placing on emerging events. This in turn may influence readers, as it determines their own focus when consuming news headlines across sources (e.g., Iyengar \& Simon, 1993).

CogMedia can be used to explore the dynamics of intermedia attention (Yerkes \& Dale, in preparation). Time stamps on the release of stories on feeds permit analysis of these dynamics, assessing how multiple outlets may converge on news themes (cf. dynamics: Wells et al., 2019). This could also reveal influence patterns across outlets. For example, in initial qualitative analysis of attention on CogMedia, the New York Times seems in particular to have influence on story themes or topics, consistent with prior observations (McCombs \& Reynolds, 2009; but see Vargo \& Guo, 2017 for some recent nuanced analysis 


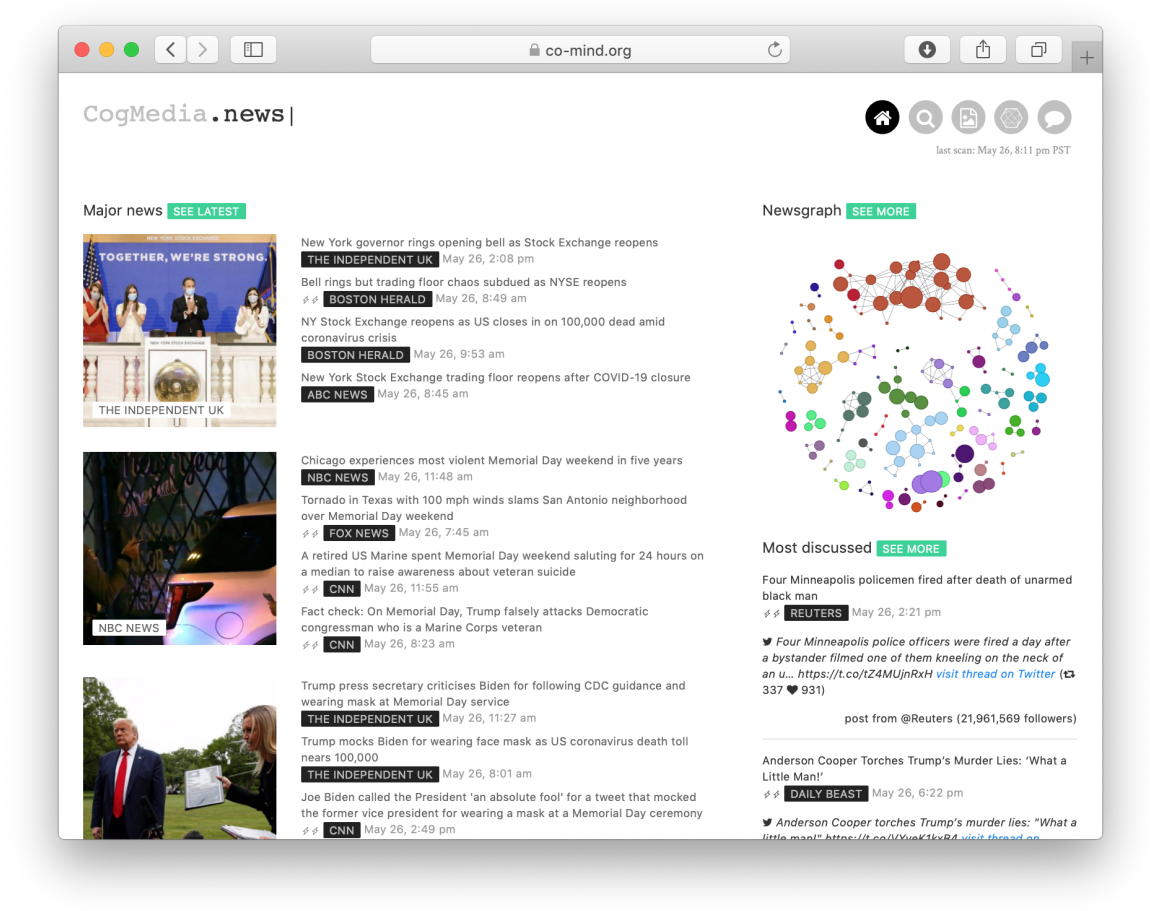

Figure 4: An illustration of the generic headline platform for news consumption (May 26th, 2020, evening). Some basic algorithms from CogMedia analysis are used for thematic organization and search, permitting users to consume the news, and researchers to study this consumption. The current dashboard can be viewed here: co-mind.org/cogmedia/browse

using causal methods).

To do this analysis, the author and collaborators are using a simple graph-theoretic method to determine the presence of news themes. Across 12 hours, news stories are interconnected if they share what is called content words. Content words are contrasted with closed-class or function words, like prepositions and determiners and so on. After filtering out closed-class words, stories are then interconnected if they share three of the same content words. Doing this produces a "newsgraph" of multi-outlet coverage. This is shown in Fig. 3. Each node in this graph is a story, and connections reflect shared content words. ${ }^{7}$ The size of the nodes is proportional to the social media score (soc) described above.

This method offers a visualization for rapidly scanning news headlines, and so has been included in the dashboarding and consumption dynamics CogMedia tools (see below). But it also provides a visual basis for assessing intermedia structure. And because time stamps are tagged on stories, it is possible to analyze the emergence of these networks, assess which news outlets seem to initiate such convergence, and determine how coverage then dissipates.

\subsection{Consumption dynamics}

The CogMedia framework also includes a platform for presenting news stories and images in the browser. The main CogMedia system includes a generic platform for major headlines, but it can also be rapidly adapted for specific purposes (see below regarding dashboarding). An illustration of the main CogMedia outreach platform is shown in Fig. 4.

This platform is also architected for behavioral research. The present setup does not yet deploy this feature, and does not track visitors. But it is possible to include tracks of reader consumption from story to story, to determine which are visited, which may be shared, how search is conducted and so on. These are standard features of enterprise news systems, but

${ }^{7}$ The text data used to build the network diagrams in Fig. 3 are based on a simple three-word overlap algorithm. This could be significantly enhanced by using more advanced natural language processing, as illustrated in the application of document indexing methods in Nicholls (2019). 


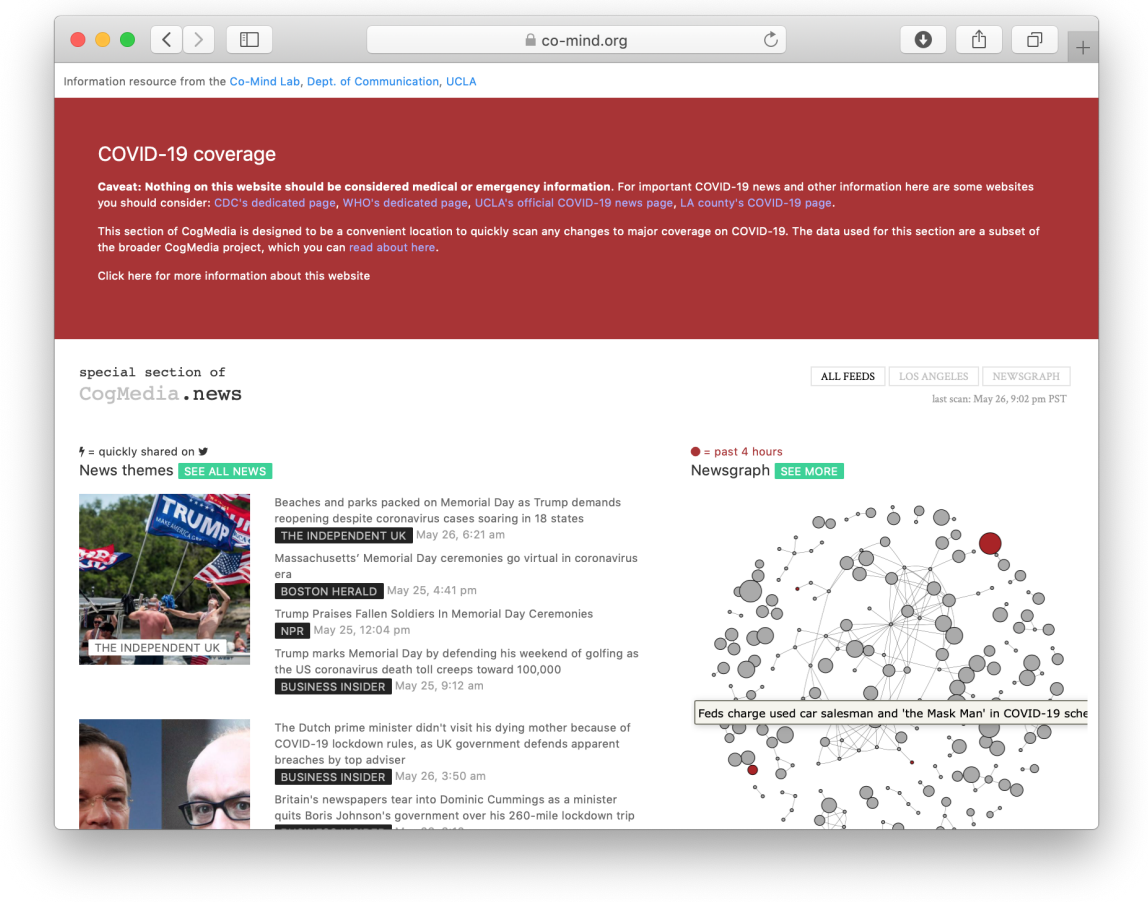

Figure 5: From the evening of May 26th, 2020. CogMedia database is filtered by chosen themes (in this case, the COVID pandemic). The newsgraph marks stories that are widely shared on social media (the size of the dot) and the relative recency (new stories are in red). The bespoke COVID-19 dashboard can be viewed here: co-mind.org/cogmedia/browse/covid.php

in CogMedia they can become a platform for experimental control of consumption tracking in a manner similar to methods used in selective exposure research (e.g., Iyengar \& Hahn, 2009; Knobloch-Westerwick \& Meng, 2009).

For example, the platform presently has the means to track comments by users. These comments are currently anonymized using a nonce naming scheme. But comments may also be a kind of consumption framework to explore the way in which news is tracked and discussed. There is precedent for this, such as in studies of news selectivity biased by partisan association (e.g., Iyengar \& Hahn, 2009). As part of an integrated system CogMedia may permit experimental control and observation of a range of parameters simultaneously.

Critically, these big-data traces are of major ethical concern (Couldry \& Yu, 2018). Any such use of CogMedia would need to accompany carefully designed consent procedures, such as an explicit opt-in procedure with institutional oversight. After such oversight, though, it may be possible to recruit participants online, and investigate their consumption behavior as part of an experimentally controllable system.

\subsection{Bespoke dashboarding}

As illustrated in the prior section, the CogMedia database is designed in such a way that it can form the basis of an outreach or news consumption platform. As a readily manipulable database of news items, it can also be rapidly redeployed for specific purposes or designs. In Fig. 5, an example of a "bespoke dashboard" is illustrated. This illustration is based on the COVID pandemic at the time of this writing, in the spring of 2020.

At the onset of the crisis in the US, CogMedia was redeployed into a new dashboard devoted to COVID-19. The dashboard is an "at-a-glance" tool, using the same automatic algorithms and data, filtered for virus-related topics. With hundreds of thousands of stories, CogMedia contains a record of how news coverage has evolved, along with incoming coverage by the hundreds or thousands of stories per day.

For example, one resource is a kind of analysis on news stories referred to above as a 
newsgraph. Stories that are related in their language, such as the words they use, can be interconnected into a diagram. This network diagram quickly reveals "clusters" of news stories and themes, showing major emerging trends in coverage of the crisis. The network marks stories that are widely shared on social media (the size of the dot) and the relative recency (newest stories are in red).

Integrating news coverage with other data may be useful as a means of exploring how news consumption resonates with other information. A specific Los Angeles dashboard was added here, shown in the link above the newsgraph. On this regional subsection, Google's API and case lists from LA Public Health offer an animated map of positive test results across the region. In addition, it includes results to prominent news items from the Los Angeles Times and a Twitter feed from LA Public Health itself.

The prior section on consumption dynamics may be combined with deployment of bespoke dashboards to explore how consumer behavior may depend on news topic, how emerging or dissipating news trends alter consumption, how partisan affiliation may alter news selection (e.g., Iyengar \& Hahn, 2009; Knobloch-Westerwick \& Meng, 2009), and so on.

\section{Discussion and theoretical implications}

Media companies produce content through the decisions and actions of individual stakeholders. Their audiences individually choose what to consume and share. Traditional theory in mass communication abstracts over these individual processes and seeks general principles (Bryant \& Miron, 2004). Many still prominent theories, such as cultivation theory (Gerbner, 1998), originally framed the consumer as shaped by the "independent variable" of television and related media (Slater, 2007; Valkenburg, Peter, \& Walther, 2016). Under this theory, the apparent machinations of state and media companies shape the social understanding and expectations of individuals. Cultivation describes "...the independent contributions television viewing makes to viewer conceptions of social reality." (Gerbner, 1998, p. 180) The search for empirical support of these broad theories has been as central as the theorizing itself (Valkenburg et al., 2016).

With many thousands of channels, stations, websites and platforms to choose from now, the present media context complicates this description. Audiences can fine-tune their consumption, and media companies face more complex demographic tapestries (Castells, 2007; Groeling, 2013; Knobloch-Westerwick \& Meng, 2009; Morgan, Shanahan, \& Signorielli, 2014; Valkenburg et al., 2016). Moreover, the consumer can no longer be simplified as a passive recipient of media (Castells, 2007; Skalski, Neuendorf, \& Cajigas, 2017). Media platforms can rapidly adapt to the nuances of selective consumption (cf. Steen \& Dale, in preparation), and it may be feasible to do so in real time (e.g., Chang et al., 2017; Fusaroli et al., 2015). This kind of "dialogue" between companies and their consumers has become much more dynamic. Unlike much passive "television-era" theorizing, consumers and media companies are now in a kind of interplay. Beyond media "cultivation," this new interplay could be thought of as a kind of mutual cultivation: Producers and consumers now exhibit much more co-construction of the social reality through real-time engagement and experimentation in Internet and social media (Beer, 2009; Loecherbach \& Trilling, 2020; Rieder, Matamoros-Fernández, \& Coromina, 2018; Varian, 2014; Valkenburg et al., 2016). Some researchers have sought to revise and extend traditional theories in this new context, asserting their continued relevance (Morgan et al., 2015).

Some theorists have remarked on the value of new data-driven techniques to better test theory and uncover underlying cause-effect relationships. For example, Morgan and colleagues (2015) remark that "new forms of measurement" will be made possible by the synergy between digital media and the data records they produce. "Digital traces" are also included as a cornerstone of emerging computational communication science methods in Hilbert et al. (2019). As remarked by Morgan et al. (2015), some domains of mass communication theory have made use of similar resources, such as agenda-setting theory (e.g., recently: McCombs, Shaw, \& Weaver, 2014; Neuman et al., 2014; Vargo \& Guo, 2017). Indeed, it is worth observing that in the areas of corpus linguistics and natural language processing, the sheer wealth of mass media data has been under analysis for two decades or more (Masand, Linoff, \& Waltz, 1992; D. D. Lewis, 1992; K. S. Jones, 1994; Franzosi, 1995; Biber, Douglas, Conrad, \& Reppen, 1998). ${ }^{8}$ Researchers in computational arenas

\footnotetext{
${ }^{8}$ In cognitive science over three decades ago, the big-data resource TalkBank(.org) began as the Child Language
} 
have tended to focus on linguistic patterns and algorithm design. This may explain the slow import of their methods into some areas of communication, where the communication strategy and outcome of these processes are the focus. What seems to be needed is a kind of rapprochement between communication theory and the well attested computational methods now on hand.

Recent research on big data and communication reveals that many are answering this call. There is an emerging "computational communication science" using large news and other media datasets to analyze content trends in automated or semi-automated ways at scale (e.g., Joo et al., 2018; Hilbert et al., 2019; Lazer et al., 2009; S. C. Lewis et al., 2013; Nicholls \& Bright, 2019; Nicholls, 2019; Pilny, McAninch, Slone, \& Moore, 2019; Soroka, Young, \& Balmas, 2015; Steen et al., 2018; van Atteveldt, Strycharz, Trilling, \& Welbers, 2019; van Atteveldt, Margolin, Shen, Trilling, \& Weber, 2019; Winter, Hughes, \& Sanders, 2019). This and related work expands the framework of content analysis to find media trends at different scales. For example, using natural language processing and graph-theoretic methods, Nicholls and Bright (2019) develop a computational approach to identifying news trends or "story chains," to help understand how news content is thematically organized over time. Analyses of news media activity can then rely less on content analysis of smaller subsets of stories (though content analysis remains important and may enhance large-scale data analysis: S. C. Lewis et al., 2013). Theories hanker for new data and new methods; these researchers have developed frontier techniques for analyzing mass media at various scales and in various contexts.

The present paper contributes to this approach. The Cognition and Media ("CogMedia") project combines large-scale data on major news headlines and applied natural language processing. Tools based on natural language processing can index cognitive factors associated with news headlines. These indices describe the ease (or difficulty) with which a human mind may process the language of a news headline. In order to understand the linkages between these cognitive-level variables and mass media patterns, CogMedia uses an algorithm based on Twitter to mark how quickly a news story is spreading soon after its release. The data and tools in CogMedia are publicly accessible through API access, and also include a media outreach interface that permits bespoke media presentation and visualization.

\subsection{Expanding theory}

Classic theory in mass communication frames it as a system emerging from the coordination of various stakeholders. For example, in classic cultivation theory, mass communication emerges from state and corporate control of communication media and technology. These mass media may shape the social identity and behavior of entire communities of consumers. Devising rigorous quantitative studies from these theories has been a major goal of a scientific approach to mass communication systems. In the example of classic cultivation theory once again, Gerbner and collaborators over decades focused on violence in mass media as an influence on individuals and communities through surveys and content analysis (e.g., Gerbner, 1998).

Beyond cultivation, in general terms, the rich and varied theoretical landscape of mass communication and media theory may be sharply influenced by the still emerging trends in large-scale data and computational methodology. This emerging methodological influence may be particularly valuable because individual-level effects of mass media may be best understood as indirect effects, filtered through cognitive processes and social contexts of consumers (see thorough discussion in Valkenburg et al., 2016). Indirect effects may be driven by various processes - from moderator variables to interactions to subtle latent constructs that may underlie media effects - and they sometimes require richer datasets and greater statistical power to detect them.

This paper began by motivating an important next step in such theorizing, whether in mass communication or in the domain of media theory, by leveraging new data sources and techniques (Hilbert et al., 2019). These next steps are important for the present context. The causal flows in this century have become bidirectional. Indeed, flows of influence may cut across the wider set of participants in mass communication and media: editors,

Data Exchange System (CHILDES), a repository for large-scale data sharing and access, now containing many thousands of interpersonal transcriptions of child, adult and clinical contexts: MacWhinney and Snow (1985); MacWhinney (2007). These data pertain most to the interpersonal level of analysis. 
writers, advertisers and other media filters, and indeed consumers. The flows of influence now derive from all members in multiple complex patterns, perhaps better described as multiplex rather than merely bidirectional. The start of this paper introduced basic ideas from complex systems as a means to tap into this process of mutual influence. What remains is to devise data sources and other resources for testing the relevance and substance of these relationships.

The goal underlying CogMedia is to link cognitive measures to those of mass media. The broader theoretical goal is to understand how these domains are participating in a kind of complex dynamic system themselves. This problem of linking across domains is a fundamental challenge in all science. A classic approach to this problem is reductionism - urging the scientist to reduce phenomena of interest to the smallest measurable grain size. In this case, a theorist might seek to reduce mass media activity purely as a function of its individual producers and consumers. An opposing approach is emergentism, which encourages finding distinct and to some extent independent emergent principles across levels of organization. In this view, the collective actions of news producers and consumers create new regularities at the collective scale, and these cannot be reduced without losing information.

A compelling rapprochement between reductionism and emergentism has also been described in McCauley and Bechtel (2001). They develop a kind of heuristic for identifying linkages across levels. Under this theory, one might find relevant processes at the mass scale that can be identified as deriving in some sense from the cognitive level (e.g., fluency, attention, etc.). The analyses described in the previous section, among other possible uses of these data, could test these possibilities. For example, intermedia attention might exhibit dynamics that resemble the attention of an individual cognitive agent (e.g., inhibition of return, stimulus salience, etc.). A researcher might then be able to identify how attentional processes of consumers end up being reflected at the mass scale.

This sort of connection between cognitive science is readily attested in the literature on communication and the effects of media exposure. Extensive work has been done on how fundamental social and cognitive processes modulate the effects of media (e.g., Shrum, 2001). In addition, high-level cognitive processes such as mental models and narrative interpretation might contribute to an understanding of how media is engaged and interpreted (e.g., Busselle \& Bilandzic, 2008). These cognitive connections can be considered critical causal forces in media psychology (Morgan \& Shanahan, 2010), and a thorough recent canvassing of such connections can be found in Valkenburg et al. (2016)

A topic of recent study in cognitive science itself is identifying the causal wiring connecting individuals and larger-scale systems. In an elegant review of this domain, Hawkins, Goodman, and Goldstone (2019) describe research with human experimentation and computational modeling that links agent-level processes to population-level parameters, such as social network structure. These factors play a critical role in emerging social conventions, and articulating the causal flows emanating from the cognitive agents and from the structuring influence of a group's configuration (see also Goldstone, Wisdom, Roberts, \& Frey, 2013). For example, Mason, Jones, and Goldstone (2008) show that social network configuration predicts how quickly a group of participants finds a solution to a simple numerical computer game. More information (more connectivity) is not always superior, and small-world connectivity, with mostly local but some long-range connections, shows faster solutions on more difficult problems. There is some evidence that this relationship between cognitive agents and their social environments is deeply rooted, even in the emergence of human linguistic systems in general. Social configuration may predict, in part, the structure of a community's language (Lupyan \& Dale, 2015, 2016). A recent treatment of these issues by Spivey (2020) suggests that integrating these scales, from the neurocognitive to the social and beyond, may be critical for understanding a cognitive agent's focal sense of self.

The review here and in the introduction suggests that measuring and modeling interactions across scales are critical for a fuller understanding of complex systems. Mass communication systems may serve as fruitful domains in which to further test this idea. These tests would expand models of multiscale systems; they would also offer new insights into the specific and relevant domains of mind and media. The CogMedia project is designed to contribute to these aims. 


\section{Acknowledgements}

Thanks to Michael Spivey, Alexandra Paxton, Nicholas Duran and David Vinson for their feedback at various stages of this project, including suggestions about the CogMedia data and interface.

\section{References}

Althaus, S., Bajjalieh, J., Carter, J. F., Peyton, B., \& Shalmon, D. A. (2020). Cline Center Historical Phoenix Event Data. Retrieved from https://databank.illinois.edu/ datasets/IDB-2796521

Balota, D. A., Yap, M. J., Hutchison, K. A., Cortese, M. J., Kessler, B., Loftis, B., ... Treiman, R. (2007). The english lexicon project. Behavior research methods, 39 (3), $445-459$.

Bar-Yam, Y. (2004). A mathematical theory of strong emergence using multiscale variety. Complexity, 9(6), 15-24.

Bechtel, W. (2017). Explicating top-down causation using networks and dynamics. Philosophy of Science, $84(2), 253-274$.

Beer, D. (2009). Power through the algorithm? participatory web cultures and the technological unconscious. New Media \& Society, 11(6), 985-1002.

Biber, D., Douglas, B., Conrad, S., \& Reppen, R. (1998). Corpus linguistics: Investigating language structure and use. Cambridge University Press.

Bryant, J., \& Miron, D. (2004). Theory and research in mass communication. Journal of communication.

Busselle, R., \& Bilandzic, H. (2008). Fictionality and perceived realism in experiencing stories: A model of narrative comprehension and engagement. Communication Theory, 18(2), 255-280.

Carr, N. (2020). The shallows: What the internet is doing to our brains (2nd ed.). WW Norton \& Company.

Castells, M. (2007). Communication, power and counter-power in the network society. International journal of communication, 1(1), 29.

Chang, S., Zhang, Y., Tang, J., Yin, D., Chang, Y., Hasegawa-Johnson, M. A., \& Huang, T. S. (2017). Streaming recommender systems. In Proceedings of the 26th international conference on world wide web (pp. 381-389).

Chemero, A. (2011). Radical embodied cognitive science.

Contreras Kallens, P. A., Dale, R., \& Smaldino, P. E. (2018). Cultural evolution of categorization. Cognitive Systems Research, 52, 765-774.

Couldry, N., \& Yu, J. (2018). Deconstructing datafication's brave new world. New Media \& Society, 20(12), 4473-4491.

Dale, R., Fusaroli, R., Duran, N. D., \& Richardson, D. C. (2013). The self-organization of human interaction. In Psychology of learning and motivation (Vol. 59, pp. 43-95). Elsevier.

Davies, M. (2009). The 385+ million word corpus of contemporary american english (19902008+): Design, architecture, and linguistic insights. International Journal of Corpus Linguistics, 14 (2), 159-190.

Franzosi, R. (1995). Computer-assisted content analysis of newspapers. Quality and Quantity, 29(2), 157-172.

Fusaroli, R., Perlman, M., Mislove, A., Paxton, A., Matlock, T., \& Dale, R. (2015). Timescales of massive human entrainment. PloS one, 10(4).

Gerbner, G. (1998). Cultivation analysis: An overview. Mass communication and society, $1(3-4), 175-194$.

Goldstone, R. L., \& Janssen, M. A. (2005). Computational models of collective behavior. Trends in cognitive sciences, 9(9), 424-430.

Goldstone, R. L., \& Lupyan, G. (2016). Discovering psychological principles by mining naturally occurring data sets. Topics in cognitive science, 8(3), 548-568.

Goldstone, R. L., Wisdom, T. N., Roberts, M. E., \& Frey, S. (2013). Learning along with others. In Psychology of learning and motivation (Vol. 58, pp. 1-45). Elsevier.

Griffiths, T. L. (2015). Manifesto for a new (computational) cognitive revolution. Cognition, $135,21-23$. 
Groeling, T. (2013). Media bias by the numbers: Challenges and opportunities in the empirical study of partisan news. Annual Review of Political Science, 16.

Groeling, T., Joo, J., Lie, W., \& Steen, F. (2016). Visualizing presidential elections. In Annual meeting of the american political science association, philadelphia.

Gureckis, T. M., \& Goldstone, R. L. (2009). How you named your child: Understanding the relationship between individual decision making and collective outcomes. Topics in Cognitive Science, 1(4), 651-674.

Haken, H. (1987). An approach to self-organization. Self-organizing systems: The emergence of order, 417-437.

Hawkins, R. X., Goodman, N. D., \& Goldstone, R. L. (2019). The emergence of social norms and conventions. Trends in cognitive sciences, 23(2), 158-169.

Hilbert, M., Barnett, G., Blumenstock, J., Contractor, N., Diesner, J., Frey, S., .. o others (2019). Computational communication science: A methodological catalyzer for a maturing discipline. International Journal of Communication, 13, 3912-3934.

Isbilen, E. S., \& Christiansen, M. H. (2020). Chunk-based memory constraints on the cultural evolution of language. Topics in Cognitive Science, 12(2), 713-726.

Iyengar, S., \& Hahn, K. S. (2009). Red media, blue media: Evidence of ideological selectivity in media use. Journal of communication, 59(1), 19-39.

Iyengar, S., \& Simon, A. (1993). News coverage of the gulf crisis and public opinion: A study of agenda-setting, priming, and framing. Communication research, 20(3), $365-383$.

Jones, K. S. (1994). Natural language processing: a historical review. In Current issues in computational linguistics: in honour of don walker (pp. 3-16). Springer.

Jones, M. N. (2016). Big data in cognitive science. Psychology Press.

Joo, J., Steen, F. F., \& Turner, M. (2017). Red hen lab: Dataset and tools for multimodal human communication research. KI-Künstliche Intelligenz, 31(4), 357-361.

Joo, J., Steinert-Threlkeld, Z. C., \& Luo, J. (2018). Social and political event analysis based on rich media. In Proceedings of the 26th acm international conference on multimedia (pp. 2093-2095).

Juarrero, A. (2009). Top-down causation and autonomy in complex systems. In Downward causation and the neurobiology of free will (pp. 83-102). Springer.

Kelso, J. S., \& Engstrom, D. A. (2006). The complementary nature. MIT press.

Kernell, G., Lamberson, P., \& Zaller, J. (2018). Market demand for civic affairs news. Political Communication, 35(2), 239-260.

Knobloch-Westerwick, S., \& Meng, J. (2009). Looking the other way: Selective exposure to attitude-consistent and counterattitudinal political information. Communication Research, 36(3), 426-448.

Laughlin, R. B., Pines, D., Schmalian, J., Stojković, B. P., \& Wolynes, P. (2000). The middle way. Proceedings of the National Academy of Sciences, 97(1), 32-37.

Lazer, D., Pentland, A. S., Adamic, L., Aral, S., Barabasi, A. L., Brewer, D., ... others (2009). Life in the network: the coming age of computational social science. Science (New York, NY), 323(5915), 721.

Leetaru, K., \& Schrodt, P. A. (2013). Gdelt: Global data on events, location, and tone, 1979-2012. In Isa annual convention (Vol. 2, pp. 1-49).

Lewis, D. D. (1992). An evaluation of phrasal and clustered representations on a text categorization task. In Proceedings of the 15th annual international acm sigir conference on research and development in information retrieval (pp. 37-50).

Lewis, S. C., Zamith, R., \& Hermida, A. (2013). Content analysis in an era of big data: A hybrid approach to computational and manual methods. Journal of broadcasting $\&$ electronic media, 57(1), 34-52.

Loecherbach, F., \& Trilling, D. (2020). 3bij3-developing a framework for researching recommender systems and their effects. Computational Communication Research, 2(1), $53-79$.

Luna, J. M., Alegria, O., \& Dale, R. (2020). Cognitive fluency and the spread of news on social media. Poster presented at the Annual Meeting of the Cognitive Science Society.

Lupyan, G., \& Dale, R. (2015). The role of adaptation in understanding linguistic diversity. Language structure and environment: Social, cultural, and natural factors, 287-16.

Lupyan, G., \& Dale, R. (2016). Why are there different languages? the role of adaptation in linguistic diversity. Trends in cognitive sciences, 20(9), 649-660. 
MacWhinney, B. (2007). The talkbank project. In Creating and digitizing language corpora (pp. 163-180). Springer.

MacWhinney, B., \& Snow, C. (1985). The child language data exchange system. Journal of child language.

Masand, B., Linoff, G., \& Waltz, D. (1992). Classifying news stories using memory based reasoning. In Proceedings of the 15th annual international acm sigir conference on research and development in information retrieval (pp. 59-65).

Mason, W. A., Jones, A., \& Goldstone, R. L. (2008). Propagation of innovations in networked groups. Journal of Experimental Psychology: General, 137(3), 422.

McCauley, R. N., \& Bechtel, W. (2001). Explanatory pluralism and heuristic identity theory. Theory \& Psychology, 11(6), 736-760.

McCombs, M. E., \& Reynolds, A. (2009). How the news shapes our civic agenda. In Media effects (pp. 17-32). Routledge.

McCombs, M. E., Shaw, D. L., \& Weaver, D. H. (2014). New directions in agenda-setting theory and research. Mass communication and society, 17(6), 781-802.

Meadows, D. H. (2008). Thinking in systems: A primer. chelsea green publishing.

Mitchell, M. (2009). Complexity: A guided tour. Oxford University Press.

Mohammad, S. M., \& Turney, P. D. (2013). Crowdsourcing a word-emotion association lexicon. Computational Intelligence, 29(3), 436-465. Retrieved from https:// onlinelibrary.wiley.com/doi/abs/10.1111/j.1467-8640.2012.00460.x doi: 10 $.1111 / \mathrm{j} .1467-8640.2012 .00460 . \mathrm{x}$

Morgan, M., \& Shanahan, J. (2010). The state of cultivation. Journal of Broadcasting \& Electronic Media, 54(2), 337-355.

Morgan, M., Shanahan, J., \& Signorielli, N. (2014). Cultivation theory in the twenty-first century. The Handbook of Media and Mass Communication Theory, 480-497.

Morgan, M., Shanahan, J., \& Signorielli, N. (2015). Yesterday's new cultivation, tomorrow. Mass Communication and Society, 18(5), 674-699.

Neuman, W. R., Guggenheim, L., Jang, S. M., \& Bae, S. Y. (2014). The dynamics of public attention: Agenda-setting theory meets big data. Journal of Communication, 64(2), $193-214$.

Nicholls, T. (2019). Detecting textual reuse in news stories, at scale. International Journal of Communication, 13.

Nicholls, T., \& Bright, J. (2019). Understanding news story chains using information retrieval and network clustering techniques. Communication Methods and Measures, 13(1), 43-59.

Odling-Smee, F. J., Laland, K. N., \& Feldman, M. W. (2013). Niche construction: the neglected process in evolution (mpb-37) (Vol. 37). Princeton university press.

Oppenheimer, D. M. (2008). The secret life of fluency. Trends in cognitive sciences, 12(6), $237-241$.

Paxton, A., \& Griffiths, T. L. (2017). Finding the traces of behavioral and cognitive processes in big data and naturally occurring datasets. Behavior Research Methods, 49(5), 1630-1638.

Pilny, A., McAninch, K., Slone, A., \& Moore, K. (2019). Using supervised machine learning in automated content analysis: An example using relational uncertainty. Communication Methods and Measures, 13(4), 287-304.

Port, R. F., \& Van Gelder, T. (1995). Mind as motion: Explorations in the dynamics of cognition.

Reber, R., Schwarz, N., \& Winkielman, P. (2004). Processing fluency and aesthetic pleasure: Is beauty in the perceiver's processing experience? Personality and social psychology review, 8(4), 364-382.

Richardson, M. J., \& Chemero, A. (2014). Complex dynamical systems and embodiment. The Routledge handbook of embodied cognition, 39-50.

Rieder, B., Matamoros-Fernández, A., \& Coromina, Ò. (2018). From ranking algorithms to 'ranking cultures' investigating the modulation of visibility in YouTube search results. Convergence, 24(1), 50-68.

Schrodt, P. A. (2010). Automated production of high-volume, real-time political event data. In Apsa 2010 annual meeting paper.

Sherry, J. L. (2015). The complexity paradigm for studying human communication: A summary and integration of two fields. Review of Communication Research, 3, 2254 . 
Shrum, L. J. (2001). Processing strategy moderates the cultivation effect. Human Communication Research, 27(1), 94-120.

Silberstein, M., \& Chemero, A. (2012). Complexity and extended phenomenologicalcognitive systems. Topics in cognitive science, 4(1), 35-50.

Silge, J., \& Robinson, D. (2016). tidytext: Text mining and analysis using tidy data principles in r. Journal of Open Source Software, 1(3), 37.

Skalski, P. D., Neuendorf, K. A., \& Cajigas, J. A. (2017). Content analysis in the interactive media age. The content analysis guidebook, 2, 201-42.

Slater, M. D. (2007). Reinforcing spirals: The mutual influence of media selectivity and media effects and their impact on individual behavior and social identity. Communication theory, 17(3), 281-303.

Soroka, S., Young, L., \& Balmas, M. (2015). Bad news or mad news? sentiment scoring of negativity, fear, and anger in news content. The ANNALS of the American Academy of Political and Social Science, 659(1), 108-121.

Spivey, M. J. (2008). The continuity of mind. Oxford University Press.

Spivey, M. J. (2020). Who you are: The science of connectedness. MIT Press.

Steen, F. F., \& Dale, R. (in preparation). Communicative potential. TBD.

Steen, F. F., Hougaard, A., Joo, J., Olza, I., Cánovas, C. P., Pleshakova, A., ... others (2018). Toward an infrastructure for data-driven multimodal communication research. Linguistics Vanguard, 4(1).

Streeck, J., \& Jordan, J. S. (2009). Communication as a dynamical self-sustaining system: the importance of time-scales and nested context. Communication Theory, 19(4), $445-464$.

Tandoc Jr, E. C., \& Foo, C. Y. W. (2018). Here's what buzzfeed journalists think of their journalism. Digital Journalism, 6(1), 41-57.

Tandoc Jr, E. C., \& Jenkins, J. (2017). The buzzfeedication of journalism? how traditional news organizations are talking about a new entrant to the journalistic field will surprise you! Journalism, 18(4), 482-500.

Thelen, E., \& Smith, L. B. (1996). A dynamic systems approach to the development of cognition and action. MIT press.

Turvey, M. T. (1990). Coordination. American psychologist, 45(8), 938.

Turvey, M. T. (2004). Impredicativity, dynamics, and the perception-action divide. In Coordination dynamics: Issues and trends (pp. 1-20). Springer.

Uscinski, J. E. (2009). When does the public's issue agenda affect the media's issue agenda (and vice-versa)? developing a framework for media-public influence. Social Science Quarterly, 90(4), 796-815.

Valkenburg, P. M., Peter, J., \& Walther, J. B. (2016). Media effects: Theory and research. Annual review of psychology, 67, 315-338.

van Atteveldt, W., Margolin, D., Shen, C., Trilling, D., \& Weber, R. (2019). A roadmap for computational communication research. Computational Communication Research, $1(1), 1-11$.

van Atteveldt, W., Strycharz, J., Trilling, D., \& Welbers, K. (2019). Toward open computational communication science: A practical road map for reusable data and code. International Journal of Communication (19328036), 13.

Van Orden, G., \& Stephen, D. G. (2012). Is cognitive science usefully cast as complexity science? Topics in cognitive science, 4(1), 3-6.

Vargo, C. J., \& Guo, L. (2017). Networks, big data, and intermedia agenda setting: An analysis of traditional, partisan, and emerging online us news. Journalism \& Mass Communication Quarterly, 94(4), 1031-1055.

Varian, H. R. (2014). Beyond big data. Business Economics, 49(1), 27-31.

Ward, M. D., Beger, A., Cutler, J., Dickenson, M., Dorff, C., \& Radford, B. (2013). Comparing gdelt and icews event data. Analysis, 21(1), 267-97.

Wells, C., Shah, D. V., Pevehouse, J. C., Foley, J., Lukito, J., Pelled, A., \& Yang, J. (2019). The temporal turn in communication research: Time series analyses using computational approaches. International Journal of Communication (19328036), 13.

Winter, N. J., Hughes, A. G., \& Sanders, L. M. (2019). Online coders, open codebooks: New opportunities for content analysis of political communication. Political Science Research and Methods, 1-16.

Young, L. C. (2017). List cultures: Knowledge and poetics from mesopotamia to buzzfeed. Amsterdam University Press. 